\title{
Frecuencia de la potencial interacción entre trimetoprim/sulfametoxazol y espironolactona por riesgo de hiperkalemia en pacientes colombianos
}

\author{
Frequency of the potential interaction \\ between trimethoprim / sulfamethoxazole and \\ spironolactone with risk for hyperkalemia in \\ colombian patients
}

\author{
Jorge Enrique Machado-Alba, Manuel Enrique Machado-Duque, \\ Andrés Gaviria-Mendoza, Juan Sebastián Restrepo-López, \\ Juan Felipe Benítez-Mejía • Pereira (Colombia)
}

\section{Resumen}

Introducción: se ha descrito el riesgo aumentado de muerte súbita y hospitalización por hiperkalemia en pacientes que consumen medicamentos ahorradores de potasio y trimetoprim, motivo por el cual se buscó determinar la frecuencia de la potencial interacción entre espironolactona y trimetoprim-sulfametoxazol en pacientes mayores de 60 años de Colombia.

Métodos: estudio observacional. De una base de datos de 3.6 millones de personas se seleccionaron pacientes mayores de 60 años que recibieron espironolactona de manera ambulatoria por al menos tres meses consecutivos y pacientes con prescripción de trimetoprim-sulfametoxazol entre el $1^{\circ}$ de agosto de 2014 y 31 de julio de 2015. Posteriormente se identificaron aquellos con prescripción conjunta durante un mismo mes. Se incluyeron variables sociodemográficas, uso concomitante de inhibidores de sistema renina angiotensina, diuréticos e inotrópicos.

Resultados: durante el año de estudio, se encontraron 8941 pacientes mayores de 60 años con prescripción continua de espironolactona, y 8028 pacientes con trimetoprim-sulfametoxazol. Su prescripción conjunta fue detectada en 77 pacientes ( $0.8 \%$ de pacientes con espironolactona), con una incidencia acumulada de 0.86 casos por 100 pacientes-espironolactona/año. La edad promedio de estos pacientes fue $79.1 \pm 14$ años, $57.1 \%$ fueron hombres, y la ciudad con más presentación de casos fue Cali (13\% del total). El $68.8 \%$ de los casos tuvieron además medicación concomitante con losartan y $62.3 \%$ con furosemida.

Conclusiones: la interacción entre espironolactona y trimetoprim-sulfametoxazol en una población colombiana, es relativamente poco frecuente; sin embargo, debido a los riesgos a los que se expone el paciente anciano es relevante por sus implicaciones en morbilidad y mortalidad, requiriendo ser conocida y monitoreada por el médico prescriptor. (Acta Med Colomb 2017; 42: 189-192).

Palabras clave: espironolactona; combinación trimetoprim-sulfametoxazol; hiperpotasemia; geriatría; farmacoepidemiologia (fuente DeCS).

\footnotetext{
Abstract

Introduction: The increased risk of sudden death and hospitalization due to hyperkalemia in patients consuming potassium-sparing drugs and trimethoprim has been described. Therefore, the frequency of the potential interaction between spironolactone and trimethoprim-sulfamethoxazole in patients older than 60 years of Colombia was sought.

Methods: observational study. From a database of 3.6 million people, patients older than 60 years who received spironolactone on an outpatient basis for at least three consecutive months and patients with a prescription for trimethoprim-sulfamethoxazole between 08/01/2014 and 07/31/2015
}

Dr. Jorge Enrique Machado-Alba: Profesor Titular de Farmacología y Toxicología, Facultad de Ciencias de la Salud. Director Grupo de Investigación en Farmacoepidemiología y Farmacovigilancia, Universidad Tecnológica de Pereira-Audifarma S.A.; Dres. Manuel Enrique Machado-Duque, Andrés Gaviria Mendoza: Grupo de Investigación en Farmacoepidemiología y Farmacovigilancia Universidad Tecnológica de Pereira-Audifarma S.A.; Dres. Juan Sebastián Restrepo-López, Juan Felipe Benítez-Mejía: Universidad Tecnológica de Pereira. Pereira (Colombia).

Correspondencia. Dr. Jorge Enrique MachadoAlba. Pereira (Colombia).

E-mail: machado@upt.edu.co

Recibido: 8/I/2016 Aceptado: 4/IX/2017 
were selected. Subsequently, those with joint prescription during the same month were identified. Sociodemographic variables, concomitant use of renin angiotensin system inhibitors, diuretics and inotropes were included.

Results: During the year of study, 8941 patients older than 60 years with continuous spironolactone prescription, and 8028 patients with trimethoprim-sulfamethoxazole, were found. Its coprescription was detected in 77 patients ( $0.8 \%$ of patients with spironolactone), with a cumulative incidence of 0.86 cases per 100 patients-spironolactone / year. The mean age of these patients was $79.1 \pm 14$ years, $57.1 \%$ were men, and the city with the most cases was Cali ( $13 \%$ of the total). $68.8 \%$ of the cases also had concomitant medication with losartan and $62.3 \%$ with furosemide.

Conclusions: The interaction between spironolactone and trimethoprim-sulfamethoxazole in a Colombian population is relatively infrequent; however, due to the risks to which the elderly patient is exposed, it is relevant because of its morbidity and mortality implications, requiring to be known and monitored by the prescribing physician. (Acta Med Colomb 2017; 42:189-192)

Keywords: spironolactone; trimethoprim-sulfamethoxazole combination; hyperkalemia; geriatrics; pharmacoepidemiology (DeCS source).

\section{Introducción}

La insuficiencia cardiaca (IC), afecta a $2 \%$ de la población general y $7 \%$ de ancianos, alterando considerablemente la calidad y expectativa de vida $(1,2)$. En el caso de una fracción de eyección $\leq 35 \%$ se indica el uso de espironolactona $(3,4)$, esteroide sintético que bloquea receptores de aldosterona y actúa como antihipertensivo y diurético, lo cual puede reducir la mortalidad un $30 \%$ en dos años (4). Sin embargo, puede elevar el potasio sérico, situación adversa en adultos mayores, o aquellos con insuficiencia renal, diabetes mellitus o que utilizan otros fármacos que elevan el potasio sérico $(5,6)$.

Recientemente se ha descrito el riesgo aumentado de muerte súbita y hospitalización por hiperkalemia en pacientes que consumen espironolactona y trimetoprim-sulfametoxazol, lo cual parece ser explicado por la similaridad molecular del trimetoprim con el amiloride, un diurético ahorrador de potasio con capacidad de elevar los niveles de potasio explicado por su mecanismo de acción $(7,8)$; que potencia la hiperkalemia y aumenta el riesgo de problemas del ritmo cardiaco y muerte súbita. En el año 2015 Antoniou y cols, encontraron un aumento de muerte súbita 2.46 veces mayor en usuarios de esta prescripción conjunta (9).

Se buscó determinar la frecuencia de la prescripción conjunta de espironolactona y trimetoprim-sulfametoxazol en adultos mayores de Colombia para estimar el riesgo a que están sujetos por la interacción.

\section{Material y métodos}

Estudio observacional, con sujetos mayores de 60 años afiliados al Sistema General de Seguridad Social en Salud de Colombia (SGSSS). Se seleccionaron pacientes que recibieron espironolactona de manera ambulatoria por al menos tres meses consecutivos y aquellos con al menos una prescripción de trimetoprim-sulfametoxazol entre el $1^{\circ}$ de agosto de 2014 y 31 de julio de 2015. Posteriormente, se identificaron los pacientes $>60$ años con la prescripción conjunta de estos fármacos durante un mismo mes (Figura 1).
La información de dispensación se obtuvo a partir de una base de datos poblacional perteneciente a Audifarma S.A, el mayor dispensador logístico de medicamentos de Colombia con base en Pereira, Risaralda, que cuenta con un área de farmacoepidemiología que permitió el acceso a esta información, que almacena datos de alrededor de 3.6 millones de afiliados a cinco entidades promotoras de salud (EPS). Se evaluaron las siguientes variables:

Sociodemográficas: edad, sexo, ciudad de residencia.

Farmacológicas: dosis y presentación de espironolactona, trimetoprim-sulfametoxazol prescritos.

Comedicación prescrita con potencial alteración en niveles de potasio séricos; inhibidores de enzima convertidora de angiotensina-iECA, antagonistas del receptor angiotensinaARA-II, diuréticos (tiazídicos, de asa) y digoxina.

El protocolo fue aprobado por el Comité de Bioética de la Universidad Tecnológica de Pereira en la categoría de "Investigación sin Riesgo". Se creó una base de datos en Microsoft Excel y para el análisis de los datos se utilizó SPSS 23.0 para Windows. Se empleó estadística descriptiva de frecuencias para variables continuas y proporciones para variables categóricas.

\section{Resultados}

Durante el año de observación se encontraron 8941 pacientes $>60$ años con mínimo tres meses de prescripción continua de espironolactona de cualquier presentación, con un promedio de 6463 prescripciones/mes del mismo. De trimetoprim-sulfametoxazol se hallaron 8028 pacientes con alguna prescripción durante el año con un promedio de 737 pacientes/mes.

Al buscar la prescripción conjunta en un mismo mes se encontró un total de 77 pacientes durante el periodo de seguimiento (Figura 1), estimando una incidencia acumulada de 0.86 por 100 pacientes-espironolactona/año.

Los sujetos con la prescripción conjunta de la potencial interacción tuvieron una edad promedio de 79.1ะ14 años, con predominio masculino $(57.1 \%)$, residentes en 31 ciuda- 


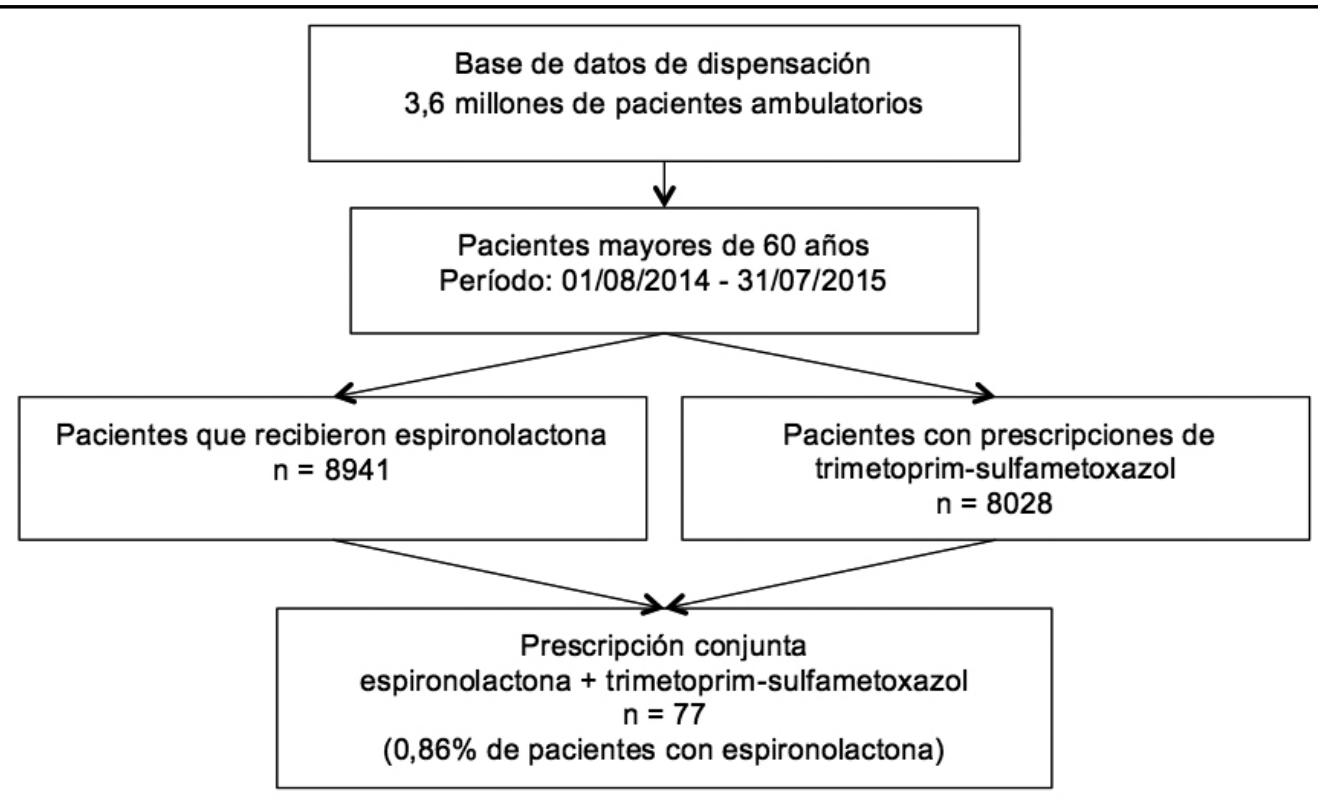

Figura 1. Diagrama de flujo sobre la identificación de los pacientes con la prescripción conjunta de espironolactona y trimetoprim-sulfametoxazol, Colombia. 2014-2015.

des de Colombia, especialmente Cali (13\% de casos), Pasto (10.4\%), Pereira (7.8\%) y Barranquilla $(6.5 \%)$.

Se encontró que al $96.9 \%$ de los pacientes se les prescribió la presentación de $25 \mathrm{mg}$ de espironolactona y todos los casos prescritos usaron la forma farmacéutica de trimetoprim 160 mg y sulfametoxazol $800 \mathrm{mg}$. Las medicaciones conjuntas en estos pacientes de interés mostraron principalmente el uso de losartan y furosemida (Tabla 1). El 71.4\% $(\mathrm{n}=55)$ de los pacientes recibió un ARA-II mientras que $16.9 \%(\mathrm{n}=13)$ tenían un iECA, indicando que el $88.3 \%$ de pacientes recibió un fármaco inhibidor del sistema renina angiotensina aldosterona.

\section{Discusión}

Es relevante informar acerca de la frecuencia de la potencial interacción de trimetoprim-sulfametoxazol y espironolactona, ya que podría suscitar muerte súbita en pacientes ancianos (9). En nuestro estudio se logró identificar la prescripción conjunta de riesgo en pacientes colombianos durante un año, con frecuencia ligeramente superior a lo reportado por Antoniou y cols, quienes determinaron este uso concomitante en $10.8 \%$ de usuarios de espironolactona durante 18 años de seguimiento ( $0.6 \%$ año), comparado con $0.86 \%$ del presente estudio, encontrándose datos similares de prescripción conjunta entre ambos grupos de pacientes, a pesar de las diferencias propias de poblaciones distintas (7).

Es claro resaltar la baja incidencia de prescripción conjunta, pudiendo explicarse por la disminución en el uso global de trimetoprim-sulfametoxazol en Colombia que entre 2005 y 2006 bajó de 14.9-13\% del total de antibióti$\cos$, mientras que los restantes, en especial las penicilinas, incrementaron $1.4 \%$ en el mismo periodo (10). Este descenso en el uso puede estar relacionado a la creciente resistencia
Tabla 1. Frecuencia de comedicaciones y combinaciones farmacológicas con potencial alteración del potasio en 77 pacientes mayores de 60 años con la interacción espironolactona + trimetoprim-sulfametoxazol, Colombia. 2014 - 2015.

\begin{tabular}{|c|c|c|}
\hline Espironolactona + trimetoprim/sulfametoxazol & $\begin{array}{c}\begin{array}{c}\text { Frecuencia } \\
(n=77)\end{array} \\
\end{array}$ & $\%$ \\
\hline Losartan & 53 & 68.8 \\
\hline Furosemida & 48 & 62.3 \\
\hline Enalapril & 12 & 15.6 \\
\hline Hidroclorotiazida & 6 & 7.8 \\
\hline Betametildigoxina & 4 & 5.2 \\
\hline Irbesartan & 1 & 1.3 \\
\hline Valsartan & 1 & 1.3 \\
\hline Captopril & 1 & 1.3 \\
\hline \multicolumn{3}{|l|}{ Esquemas - Combinaciones } \\
\hline ARA-II ${ }^{1}+$ Furosemida & 35 & 45.5 \\
\hline $\mathrm{IECA}^{2}+$ Furosemida & 9 & 11.7 \\
\hline ARA II + Hidroclorotiazida & 5 & 6.5 \\
\hline $\begin{array}{l}{ }^{1} \text { Antagonista de receptor de angiotensina } \\
{ }^{2} \text { Inhibidor de enzima convertidora de antiotensina }\end{array}$ & & \\
\hline
\end{tabular}

reportada en los últimos años, como se evidencia en estudios locales de urocultivos en los cuales Escherichia coli fue resistente en $47.9 \%$ de casos, además en Klebsiella sp y Enterococcus sp la sensibilidad no superó $80 \%$, por lo cual no se encontró un mayor cantidad de pacientes con esta prescripción $(11,12)$.

La edad promedio de los pacientes a quienes se les detectó la interacción fue cercana a los 80 años, similar a otros estudios donde la mayoría de pacientes identificados tenían entre 75 y 84 años (7) o más de 85 años (9), reforzando la 
problemática de que el grupo poblacional que está en mayor riesgo de estas interacciones y además de desarrollar un evento adverso es precisamente la población longeva (9). En este caso hubo predominio de pacientes de sexo masculino, probablemente por su mayor prevalencia de IC (1) y la necesidad de formular espironolactona. Sin embargo, difiere de la serie de Antoniou y cols, en la cual las mujeres representaron $65 \%$ de los casos (7).

La dosis de espironolactona que se describe en la literatura científica para esta interacción farmacológica ( 25 mg) $(7,9)$ es la misma que se está utilizando en nuestro medio. Cabe resaltar que en este reporte la presentación de trimetoprim-sulfametoxazol fue de tabletas de $160+800 \mathrm{mg}$, lo cual se ha asociado con mayor riesgo de hiperkalemia comparado con las prescripciones de tabletas de $80+400 \mathrm{mg}$ (OR: 13.5 vs 9.7), por lo cual es importante que los médicos prescriptores conozcan la interacción, los medicamentos y dosis que están siendo utilizados y prevengan reacciones adversas evitables (7).

En el presente estudio tanto losartan como furosemida fueron fármacos frecuentemente medicados de manera concomitante, siendo de importancia al considerar su interacción con los niveles de potasio sérico. Por un lado, furosemida puede disminuir los niveles séricos de este ion y evitar el riesgo de hiperkalemia, mientras que los iECA y ARA-II la pueden exacerbar, incluso relacionándolos con riesgo aumentado de hospitalización por dicha causa al emplearlos con trimetoprim-sulfametoxazol (13). Es necesario analizar mayor información no sólo de estos fármacos sino también de otros que no se consideraron en este estudio pero que están siendo prescritos y pudieran tornarse en factores protectores o de riesgo.

Aunque es innegable que por ahora sólo se puede hablar de un riesgo y no de causalidad (14), lo cierto es que en nuestro medio es importante considerar esta interacción y se hace necesario consolidar más estudios que permitan no sólo determinar la relación de causa-efecto, sino también informar acerca del riesgo potencial con fármacos que pudieran exacerbar el efecto elevador del potasio sérico, para mejorar las prácticas de prescripción $(15,16)$.

Entre las limitaciones de este estudio se debe considerar que la información se obtuvo de una base de datos poblacional de dispensación y no es posible determinar si los pacientes consumieron realmente los dos medicamentos, $\mathrm{o}$ si aparecieron eventos adversos, así como la imposibilidad de medir el desenlace evaluado en otros estudios como mortalidad, también es importante resaltar que fueron pacientes afiliados al SGSSS del régimen contributivo y no se pueden extrapolar los resultados a poblaciones con diferentes características o tipos de aseguramiento. Como fortalezas presenta la rigurosidad en la obtención de la información y el tamaño de la población evaluada.

En la actualidad se recomienda el monitoreo de los niveles de potasio en sangre poco tiempo después de instaurada la terapia con trimetoprim-sulfametoxazol, especialmente en pacientes ancianos con función renal disminuida, diabetes mellitus y prescripciones conjuntas de iECAs, ARA-II y espironolactona (17). Sin embargo, no es una práctica clínica utilizada en el medio colombiano.

La interacción entre espironolactona y trimetoprimsulfametoxazol en una población colombiana es relativamente poco frecuente, sin embargo debido a los riesgos a los que se expone el paciente anciano es relevante por sus implicaciones en morbilidad y mortalidad, requiriendo ser conocida y monitoreada por el médico prescriptor.

\section{Agradecimientos}

A Viviana Andrea Orozco por el apoyo en el procesamiento de los datos para obtener la base de datos inicial.

\section{Financiación}

El trabajo fue financiado por la Universidad Tecnológica de Pereira y Audifarma S.A.

\section{Conflicto de intereses}

Los autores indican que no existe ningún conflicto de intereses.

\section{Referencias}

1. Gardner RS, McDonagh TA. Chronic heart failure: epidemiology, investigation and management. Medicine. 2014; 42(10): 562-7.

2. McDonagh TA, Morrison CE, Lawrence A, Ford I, Tunstall-Pedoe H, McMurray JJV, et al. Symptomatic and asymptomatic left-ventricular systolic dysfunction in an urban population. Lancet. 1997;350(9081):829-33.

3. Pitt B. Heart failure: the role for mineralocorticoid receptor antagonists. Swiss Med Wkly. 2014; 144: w13959.

4. Pitt B, Zannad F, Remme WJ, Cody R, Castaigne A, Perez A, et al. The effect of spironolactone on morbidity and mortality in patients with severe heart failure. N Engl J Med. 1999; 341(10): 709-17.

5. Juurlink DN, Mamdani MM, Lee DS, Kopp A, Austin PC, Laupacis A, et al Rates of hyperkalemia after publication of the Randomized Aldactone Evaluation Study. N Engl J Med. 2004; 351(6): 543-51.

6. Aguirre M, Medina JE, Chacon JA, Restrepo CA. Uso de IECA o ARA II más espironolactona y su relación con hiperkalemia en pacientes ambulatorios. Acta Med Colomb 2007; 32(4): 212-218.

7. Antoniou T, Gomes T, Mamdani MM, Yao Z, Hellings C, Garg AX, et al. Trimethoprim-sulfamethoxazole induced hyperkalaemia in elderly patients receiving spironolactone: nested case-control study. BMJ. 2011; 343: d5228.

8. Wei L, MacDonald TM, Mackenzie IS. Co-prescription of co-trimoxazole and spironolactone in elderly patients. BMJ. 2011;343: d5656.

9. Antoniou T, Hollands S, Macdonald EM, Gomes T, Mamdani MM, Juurlink DN, et al. Trimethoprim-sulfamethoxazole and risk of sudden death among patients taking spironolactone. CMAJ. 2015; 187(4): E138-43.

10. Machado-Alba JE, González-Santos DM. Dispensación de antibióticos de uso ambulatorio en una población colombiana. Rev salud pública. 2009; 11(5): 734-44.

11. Machado-Alba JE, Murillo-Muñoz MM. Evaluación de sensibilidad antibiótica en urocultivos de pacientes en primer nivel de atención en salud de Pereira. Rev salud pública. 2012; 14(4): 710-9.

12. Orrego-Marin CP, Henao-Mejia CP, Cardona-Arias JA. Prevalencia de infección urinaria, uropatógenos y perfil de susceptibilidad antimicrobiana. Acta Med Colomb. 2014; 39(4): 353.

13. Antoniou T, Gomes T, Juurlink DN, Loutfy MR, Glazier RH, Mamdani MM. Trimethoprim-Sulfamethoxazole-Induced Hyperkalemia in Patients Receiving Inhibitors of the Renin-Angiotensin System: A Population-Based Study. Arch Intern Med. 2010; 170(12): 1045-9.

14. Ananthakrishnan S. ACP Journal Club. Trimethoprim-sulfamethoxazole was linked to sudden death in older patients using spironolactone. Ann Intern Med. 2015; 162(12): JC13.

15. Atkin PA, Veitch PC, Veitch EM, Ogle SJ. The Epidemiology of Serious Adverse Drug Reactions Among the Elderly. Drugs Aging. 1999; 14(2): 141-52.

16. Gnjidic D, Hilmer SN, Blyth FM, Naganathan V, Waite L, Seibel MJ, et al. Polypharmacy cutoff and outcomes: five or more medicines were used to identify community-dwelling older men at risk of different adverse outcomes. J Clin Epidemiol. 2012; 65(9): 989-95.

17. Ho JM-W, Juurlink DN. Considerations when prescribing trimethoprim-sulfamethoxazole. CMAJ. 2011; 183(16): 1851-8. 